\title{
Three-quark force in p-p elastic scattering
}

\author{
M. A. Hassan ${ }^{1, a}$, A. A. E. Hefny ${ }^{2}$, T. N. E. Salama ${ }^{1}$ \\ ${ }^{1}$ Mathematics Department, Faculty of Science, Ain Shams University, Cairo, Egypt \\ ${ }^{2}$ Basic Science Department, Faculty of Computer and Information Sciences, Ain Shams University, Cairo, Egypt
}

Received: 5 July 2018 / Accepted: 10 November 2018 / Published online: 21 December 2018

(C) The Author(s) 2018

\begin{abstract}
In the framework of Glauber optical limit approximation, considering the proton has an outer pion cloud of radius $\sim 0.87 \mathrm{fm}$ and an inner core of radius $\sim 0.44 \mathrm{fm}$ where the valence three quarks are confined, and including two-gluon exchange three-quark force, a good fit with the experimental data of $\mathrm{p}-\mathrm{p}$ elastic scattering differential cross section up to $q^{2} \approx 3(\mathrm{GeV} / \mathrm{c})^{2}$, total cross section and the ratio of real to imaginary parts of elastic scattering amplitude in the forward direction is obtained at laboratory momenta 200, 290, 500, 1070 and $1500 \mathrm{GeV} / \mathrm{c}$. The radii of two-quark interaction $r_{t}$ and three-quark force $r_{t h}$ are calculated. The quant energy representing the gluon $E_{g}$ is evaluated.
\end{abstract}

\section{Introduction}

Proton-proton scattering at very high energy taking into account the quark structure of proton located is becoming in the centre of the attention of researchers, especially with many measurements of proton-proton scattering cross sections. Different theoretical models are suggested to study different properties of proton structure and of proton-proton scattering mechanism. In most cases the phenomenological models with some physical picture of proton structure are used. Despite the availability of LHC data at very high energy [1-4], we will focus attention on the relatively low energy of FNAL and CERN-ISR data, where the centre of mass energy $\sqrt{s}=19.42-53 \mathrm{GeV}\left(p_{L}=200-1500 \mathrm{GeV} / \mathrm{c}\right)$ [5-9].

One of the most prevalent models of proton-proton elastic scattering calculations at high energy is the Regge-pole theory with the Pomeron exchange. In general, in the framework of this theory, a good agreement with the proton-proton elastic scattering differential cross section, total cross section $\sigma_{t}$ and the ratio $\rho$ of real to imaginary parts of protonproton elastic scattering amplitude in the forward direction was obtained at FNAL and CERN-ISR energy [10-14]. A similar fit with the experimental data of the same protonproton quantities at the same energy was obtained by using many different other approaches, for example, the eikonalization approach [15], Bialas-Bzdak model [16], axiomatic quantum field theory [17] and others [18-22].

At higher energies of CDF, HERA and LHC the picture of the theoretical results of proton-proton elastic scattering may be a little different and we may need a new physics [23]. With Regge-pole theory, considering a parametrization of the total cross section $\sigma_{t}$ and the ratio $\rho$ with two Reggeons and four Pomeron contributions the authors in [24] cannot obtain a good agreement with the data at $\sqrt{s}=13 \mathrm{TeV}$. However, in the framework of constituent quark model with pomeron exchange [25] a fit with the CDF and HERA data of protonproton total cross section $\sigma_{t}$ and elastic cross section $\sigma_{e}$ was improved including the triple pomeron vertex and with the double pomeron exchange and the authors in [25] show that the radius of the constituent quark $R_{q} \approx 0.0624-0.0882 \mathrm{fm}$. With the same model including all possible quark interactions and taking quark-quark scattering amplitude in eikonal approximation a good fit with experimental data of protonproton total cross section $\sigma_{t}$ and elastic cross section $\sigma_{e}$ at $\sqrt{s}=23-1855 \mathrm{GeV}$ was obtained [23]. The used quark radius to obtain the fit is $R_{q} \approx 0.0789 \mathrm{fm}$. At the same time, at $\sqrt{s}=18000 \mathrm{GeV}$ the authors found $R_{q} \approx 0.3-0.4 \mathrm{fm}$. In additive quark model with pomeron exchange approach the total cross section $\sigma_{t}$, differential cross section $\frac{d \sigma}{d t}[26]$ and the ratio $\rho$ [27] for proton-proton elastic scattering at LHC energies are calculated. In this model with only four order of interaction a good fit with data of $\frac{d \sigma}{d t}$ at $\sqrt{s}=7 \mathrm{TeV}$ was obtained up to $q^{2}=2.5(\mathrm{GeV} / \mathrm{c})^{2}$ with the quark radius $R_{q} \approx 0.44 \mathrm{fm}$. However, with the all order of quark-quark interactions the agreement with the data was observed up to $q^{2}=0.4(\mathrm{GeV} / \mathrm{c})^{2}$ only. Something must be discussed with increasing of $q^{2}$. At LHC energy, for $\sigma_{t}$ a good fit was obtained [26], while the values of the ratio $\rho$ were little below the data [27].

\footnotetext{
a e-mail: el_hamam@sci.asu.edu.eg
} 
One of the successful models in the study of proton-proton scattering as composite systems is the multiple scattering theory of Glauber [28]. Using Glauber theory [29-31] the authors show that the multiple scattering mechanism with the quark degree of freedom describes the diffraction pattern of particle-particle elastic scattering and quarks having spatial dimensions which are small compared to the corresponding hadrons and a good agreement with the experimental data of high energy was obtained. With simple Gaussian form of proton wave function, by introducing the time-ordering effect in quark-quark multi-scattering the good agreement with the proton-proton data was obtained at CERN-ISR energies [35]. Different wave functions were used in the framework of Glauber model to describe the quark distribution in the proton and obtained a good fit with the experimental data at the CERN-ISR energies [31,32]. In [32], by introducing quark-quark short rang correlations in the wave function obtained the quark radius $R_{q} \approx 0.17 \mathrm{fm}$. Using the geometrical impact parameter representation [33] obtained $R_{q} \approx 0.15 .5 \mathrm{fm}$. On the other hand, evaluations of the quark radius in the framework of Glauber theory at CERN-ISR energies are of order $R_{q} \approx 0.4 \mathrm{fm}$ [34].

Thus, approximately same value of quark radius $R_{q}$ at different energies, (CERN-ISR and LHC) may be related to different models and then different parametrization of quarkquark interaction. This interpret, also, the similar results of proton-proton cross sections in the framework of different models.

The optical limit approximation in the framework of Glauber theory is not tested for proton-proton elastic scattering. Therefore, in this work, using this approximation, we consider the physical picture of proton as used in Ref. [36], where the proton has an outer pion cloud of radius $\sim 0.87 \mathrm{fm}$ and an inner core of radius $\sim 0.44 \mathrm{fm}$ where the valence three quarks are confined. With this picture of the proton, the proton-proton elastic scattering amplitude can be written as [36]

$T(q)=T_{0}(q)+F(q)$,

where $T_{0}(q)$ represents the cloud effect, i.e., the interaction of incident proton pion cloud with the target proton pion cloud (soft Pomeron), and $F(q)$ represents the contributions of quark-quark interaction in terms of quark-quark elastic scattering amplitudes (hard Pomeron). The expressions between brackets may explain the common idea between the Pomeron approach and Glauber theory. The pion cloud has an effect in the forward direction of proton-proton scattering. In fact, since $q \sim 1 / r$, the outer cloud affects at small values of momentum transfer $q$. At the same time, for the quarks, we have two types of interactions. The first is quarkquark interaction inside the core of the proton and the other is the interaction of a quark of incident proton with a quark of target proton in the case of proton-proton scattering.
With small radius of proton core, $r_{c o r}=0.44 \mathrm{fm}$, the probability of three-quark force in proton-proton scattering cannot be neglected. However, the three-quark force was not studied. We can, simply, introduce the two-gluon exchange three-quark force effect through the profile function of quarkquark interaction in the Glauber theory in similar fashion to the used approaches in the case of $2 \pi$-exchange threenucleon force $[37,38]$. The optical limit approximation [38] is used to study three-quark force in proton-proton elastic scattering at FNAL and CERN-ISR energy. We will compare our results with the experimental data at laboratory momentum in the range $200-1500 \mathrm{GeV} / \mathrm{c}$ for the differential cross section of [5-9] and total cross section of [39,40]. We aim to obtain an information about radius of quark-quark interaction and three-quark force radius. Also, using the obtained value of two-quark force radius we aim to evaluate the quant energy representing the mediator of the force between two-quarks.

\section{Formalism}

In the optical limit approximation the elastic scattering amplitude $F(\mathbf{q})$ of two colliding protons with three-quark constitutes for each [34], including three-quark force [38] and taking into account the geometrical scaling property of protonproton reaction at CERN-ISR energies [41] is given by,

$$
F(\boldsymbol{q})=\frac{i R_{0}^{2}(s)}{2 \pi} \int d \boldsymbol{b}_{0} \exp \left\{i \boldsymbol{q}_{\boldsymbol{0}} \cdot \boldsymbol{b}_{\boldsymbol{0}}\right\}\left[1-\exp \left\{i \chi_{o p t}\left(\boldsymbol{b}_{\boldsymbol{0}}\right)\right\}\right],
$$

where $\boldsymbol{q}_{\boldsymbol{0}}=R_{\boldsymbol{0}} \boldsymbol{q}, \boldsymbol{b}_{0}=\boldsymbol{b} / R_{0}$ are dimensionless variable, $\boldsymbol{q}$ and $\boldsymbol{b}$ are the momentum transfer vector and the two- dimensional impact vector between the mass centres of the two protons, respectively. $R_{0}(s)=\sqrt{\frac{\sigma_{\text {inel }}(s)}{\pi}}$ is the inelastic radius of proton and $\sigma_{\text {inel }}(s)$ is the total inelastic cross section for proton-proton collision. In fact, the geometric scaling property is related to quark-quark collision. According to this property, the quark-quark profile function $\Gamma_{j k}\left(\boldsymbol{b}_{j k}\right)$ of scattering process is replaced by $\Gamma_{j k}\left(\boldsymbol{b}_{j k} / R_{0}\right)$, see Eqs. (4) and (7). The total optical phase shift is [38],

$$
\chi_{o p t}\left(\boldsymbol{b}_{0}\right)=\sum_{j, k} \chi_{j k}^{t}\left(\boldsymbol{b}_{0}\right)+\sum_{\substack{j, k, l \\ k \neq l}} \chi_{j(k l)}^{t h}\left(\boldsymbol{b}_{0}\right)+\sum_{\substack{j, m, k \\ j \neq m}} \chi_{(j m) k}^{t h}\left(\boldsymbol{b}_{0}\right),
$$

where $\chi_{j k}^{t}\left(\boldsymbol{b}_{0}\right)$ is the two-quark force phase shift, $\chi_{j(k l)}^{t h}\left(\boldsymbol{b}_{0}\right)$ is the three-quark force correction where the quark $k$ and quark $l$ are target quarks and $\chi_{(j m) k}^{t h}\left(\boldsymbol{b}_{0}\right)$ is the three-quark force correction where quark $j$ and quark $m$ are incident quarks. In the used approach, $j(k l)$ means that the incident quark $j$ interacts with the target quark $k$ and, at the same 
Table 1 The dimensionless two-quark force parameters $A_{0}$, $\varepsilon$ and $\beta_{0}$, three-quark force parameters $A, B$ and $\gamma_{0}$ and values of $\alpha_{0}=R_{0}^{2} /\left\langle r^{2}\right\rangle_{p}$, cloud parameters $B_{0}$ and $c_{0}$

\begin{tabular}{lllllllllll}
\hline$P_{\text {lap }}, \mathrm{GeV} / \mathrm{c}$ & $R_{0}^{2} \mathrm{mb}$ & $A_{0}$ & $\varepsilon$ & $\beta_{0}$ & $A$ & $B$ & $\gamma_{0}$ & $B_{0}$ & $c_{0}$ & $\alpha_{0}$ \\
\hline 200 & 10.19 & 1.6 & -0.153 & 0.27 & 0.22 & 0.0025 & 0.135 & 0.1147 & 0.420 & 3.0074 \\
290 & 10,30 & 1.6 & 0.050 & 0.20 & 0.33 & 0.07 & 0.100 & 0.1278 & 0.378 & 2.9332 \\
500 & 10.60 & 1.6 & 0.155 & 0.29 & 0.18 & 0.005 & 0.145 & 0.1124 & 0.404 & 3.3538 \\
1070 & 11.17 & 1.6 & 0.250 & 0.24 & 0.26 & 0.03 & 0.120 & 0.1116 & 0.383 & 3.1810 \\
1500 & 11.17 & 1.6 & 0.260 & 0.22 & 0.30 & 0.03 & 0.110 & 0.1210 & 0.418 & 2.9928 \\
Average & 10.686 & 1.6 & 0.178 & 0.244 & 0.258 & 0.0275 & 0.122 & 0.1175 & 0.4006 & 3.0936 \\
\hline
\end{tabular}

$$
\begin{aligned}
\chi_{(j m) k}^{t h}\left(\boldsymbol{b}_{0}\right)= & i \int d \boldsymbol{\rho}_{\boldsymbol{0}} d \boldsymbol{r}_{\boldsymbol{0}} d \boldsymbol{\rho}_{\boldsymbol{0}}^{\prime} d \boldsymbol{r}_{\boldsymbol{0}}^{\prime} \\
& \times\left|\varphi_{I}\left(\boldsymbol{\rho}_{\boldsymbol{0}}, \boldsymbol{r}_{\boldsymbol{0}}\right) \varphi_{T}\left(\boldsymbol{\rho}_{\boldsymbol{0}}^{\prime}, \boldsymbol{r}_{\boldsymbol{0}}^{\prime}\right)\right|^{2} \Gamma_{(j m) k}^{t h}\left(\boldsymbol{b}_{0 j k}, \boldsymbol{t}_{0 j m}\right),
\end{aligned}
$$

where $\varphi_{I}\left(\boldsymbol{\rho}_{\boldsymbol{0}}, \boldsymbol{r}_{\boldsymbol{0}}\right)$ and $\varphi_{T}\left(\boldsymbol{\rho}_{\boldsymbol{0}}^{\prime}, \boldsymbol{r}_{\boldsymbol{0}}^{\prime}\right)$ are the wave functions of the relative motion of the incident and target proton, respectively, with dimensionless variable $\left(\boldsymbol{\rho}_{0}, \boldsymbol{r}_{\boldsymbol{0}}\right)$ and $\left(\boldsymbol{\rho}_{\mathbf{0}}^{\prime}, \boldsymbol{r}_{\mathbf{0}}^{\prime}\right)$, respectively. $\rho=R_{0} \rho_{0}=\boldsymbol{r}_{3}-\left(\boldsymbol{r}_{1}+\boldsymbol{r}_{2}\right) / 2, r=R_{0} \boldsymbol{r}_{0}=\boldsymbol{r}_{1}-\boldsymbol{r}_{2}$ are the relative coordinates of quarks where $\boldsymbol{r}_{1}, \boldsymbol{r}_{2}$ and $\boldsymbol{r}_{3}$ are the position vectors of the quarks inside the proton. Also, $\boldsymbol{b}_{0 j k}=\boldsymbol{b}_{0}-\boldsymbol{t}_{0 j}+\boldsymbol{t}_{0 k}^{\prime}, \boldsymbol{t}_{0 k l}^{\prime}=\boldsymbol{t}_{0 k}^{\prime}-\boldsymbol{t}_{0 l}^{\prime}, \boldsymbol{t}_{0 j m}=\boldsymbol{t}_{0 j}-\boldsymbol{t}_{0 m} \quad \boldsymbol{t}_{01}=$ $\frac{2}{3} \boldsymbol{\rho}_{0 \perp}, \boldsymbol{t}_{02}=-\frac{1}{3} \boldsymbol{\rho}_{0 \perp}+\frac{1}{2} \boldsymbol{r}_{0 \perp}$, and $\boldsymbol{t}_{03}=-\frac{1}{3} \boldsymbol{\rho}_{0 \perp}-\frac{1}{2} \boldsymbol{r}_{0 \perp}$, where the sign $\perp$ means that the projection is on the impact plane.

The two-quark force profile function $\Gamma_{j k}^{t}\left(\boldsymbol{b}_{0}\right)$ [31] and the three-quark force profile functions $\Gamma_{j(k l)}^{t h}\left(\boldsymbol{b}_{0 j k}, \boldsymbol{t}_{0 k l}^{\prime}\right)$ and $\Gamma_{(j m) k}^{t h}\left(\boldsymbol{b}_{0 j k}, \boldsymbol{t}_{0 j m}\right)[38]$ are taken as follows

$$
\begin{aligned}
& \Gamma_{j k}^{t}\left(\boldsymbol{b}_{0 j k}\right)=\frac{A_{0 j k}}{9} \frac{\left(1-i \varepsilon_{j k}\right)}{4 \pi \beta_{0 j k}} \exp \left\{-\frac{1}{2 \beta_{0 j k}} \boldsymbol{b}_{0 j k}^{2}\right\}, \\
& \Gamma_{j(k l)}^{t h}\left(\boldsymbol{b}_{0 j k}, \boldsymbol{t}_{0 k l}^{\prime}\right)=\left(A_{j(k l)}+i B_{j(k l)}\right) \exp \left\{-\frac{1}{\gamma_{0}}\left(\boldsymbol{b}_{0 j k}{ }^{2}+\boldsymbol{t}_{0 k l}^{\prime}{ }^{2}\right)\right\},
\end{aligned}
$$

$\Gamma_{(j m) k}^{t h}\left(\boldsymbol{b}_{0 j k}, \boldsymbol{t}_{0 j m}\right)=\left(A_{(j m) k}+i B_{(j m) k}\right) \exp \left\{-\frac{1}{\gamma_{0}}\left(\boldsymbol{b}_{0 j k}^{2}+\boldsymbol{t}_{0 j m}^{2}\right)\right\}$,

where $\left(A_{0 j k}, \varepsilon_{j k}, \beta_{0 j k}\right)$ are two-quark force dimensionless parameters. The quantity $\varepsilon_{j k}$ is the ratio of the real to imaginary parts of the quark-quark amplitude in the forward direction and $\beta_{0 j k}$ is the slope parameter and is related to the radius of two-quark interaction. Note that, from the profile function $\Gamma_{j k}^{t}\left(\boldsymbol{b}_{0 j k}\right)$, we can take $r_{t}=\sqrt{2 \beta_{j k}}$ as a radius of quark-quark interaction, where $\beta_{j k}=\beta_{0 j k} R_{0}^{2}$. The parameters $\left(A_{j(k l)}, B_{j(k l)}, A_{(j m) k}, B_{(j m) k}, \gamma_{0}\right)$ are the three-quark force dimensionless parameters. Also, $r_{t h}=\sqrt{\gamma}$, where $\gamma=\gamma_{0} R_{0}^{2}$, can be considered as the three-quark force radius $[37,38]$. From the uncertainty relation of energy and time, we know that $r_{t h}=\frac{1}{2} r_{t}$. Therefore, we can write $\sqrt{\gamma}=\frac{1}{2} \sqrt{2 \beta_{j k}}=\sqrt{\frac{\beta_{j k}}{2}}$. Thus, we can take $\gamma=\frac{\beta_{j k}}{2}$.

The wave functions of incident and target protons are taken in the same Gauss form,

$\varphi(\boldsymbol{\rho}, \boldsymbol{r})=\sqrt{\frac{\alpha^{3}}{3^{3 / 2} \pi^{3}}} e^{-\frac{\alpha}{2}\left(\frac{2}{3} \rho^{2}+\frac{1}{2} r^{2}\right)}$,

where $\alpha=\frac{1}{\left\langle r^{2}\right\rangle_{p}}$ [35]. The value of $\left\langle r^{2}\right\rangle_{p}^{1 / 2}$ is taken as given in the Refs. [31,35], $\left\langle r^{2}\right\rangle_{p}^{1 / 2} \approx 0.59 \mathrm{fm}$. This value is less than the charge radius of proton, $\approx 0.87 \mathrm{fm}$. In fact, the wave functions (10) describe the motion of the three quarks inside the core of the proton. Therefore, the root mean square radius $\left\langle r^{2}\right\rangle_{p}^{1 / 2}$ in this case has a value less than the value of electromagnetic radius of the proton where the outer pion cloud is included in the measurements. In Ref. [25] the value $\left\langle r^{2}\right\rangle_{p}^{1 / 2}=0,68 \mathrm{fm}$ was used and the difference with the electromagnetic radius interpreted by the difference in the distribution of charged and strong interacting matter inside the proton.

Thus, the total proton-proton optical phase shift $\chi_{\text {opt }}$ with three-quark force correction, from Eqs. (4)-(10), is

$$
\begin{aligned}
\chi_{o p t}\left(\boldsymbol{b}_{0}\right) & =\chi_{o p t}^{t}\left(\boldsymbol{b}_{0}\right)+\chi_{o p t}^{t h}\left(\boldsymbol{b}_{0}\right) \\
& =\frac{\alpha_{0}}{12 \pi} \sum_{j, k}\left[A_{0 j k}\left(i+\varepsilon_{j k}\right) /\left(2+3 \alpha_{0} \beta_{0 j k}\right)\right]
\end{aligned}
$$


Fig. 1 The p-p elastic scattering differential cross section. The results for 200 , 290, 500, 1070 and $1500 \mathrm{GeV} / \mathrm{c}$ are given in figures $(\mathbf{a}-\mathbf{e})$, respectively. The dashed, dotdashed and solid curves represent the results of (two-quark force) only, (two-quark force + pion cloud effect), and (two-+three-quark force+ pion-cloud effect), respectively. The experimental data are taken from [5-9]
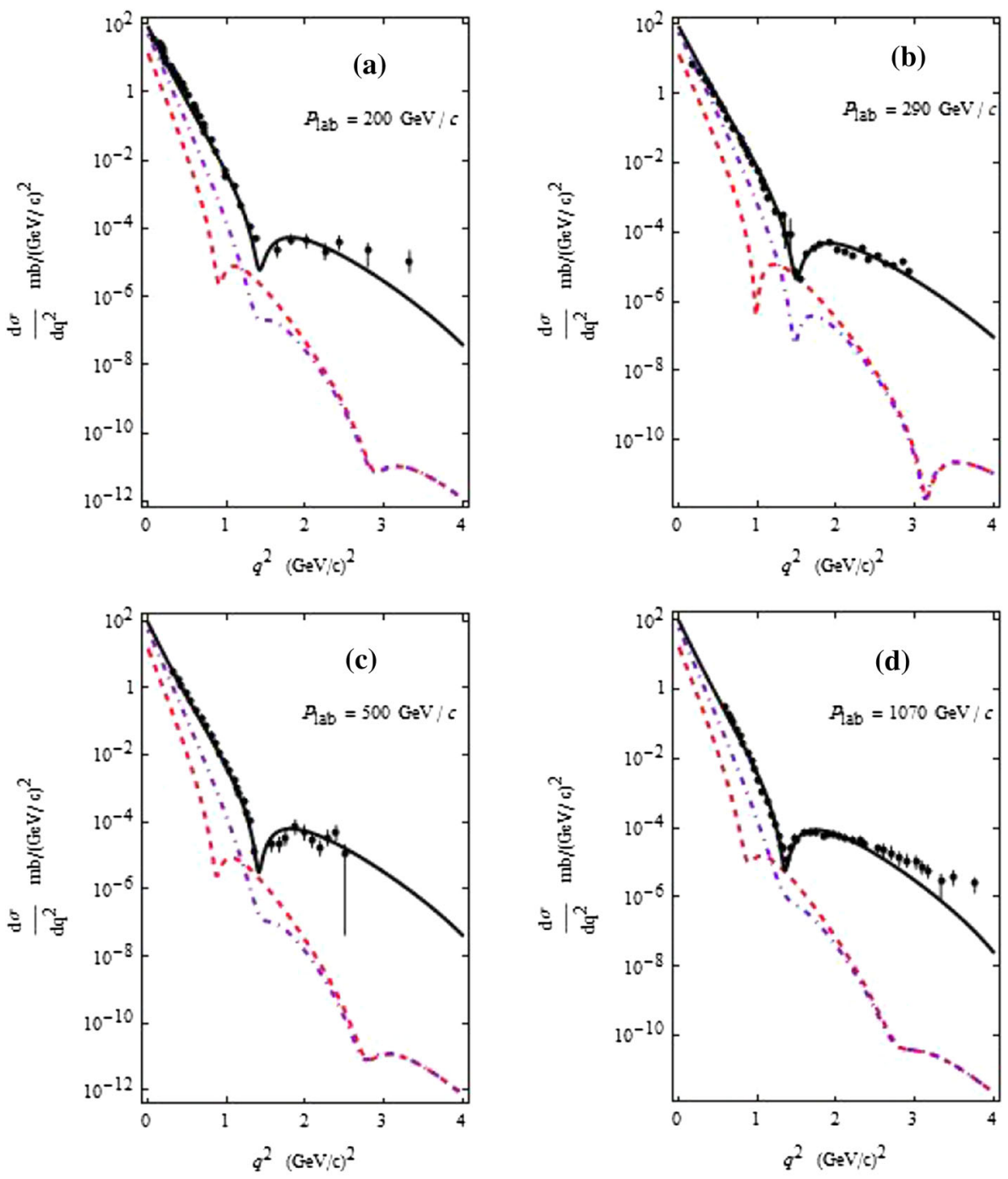

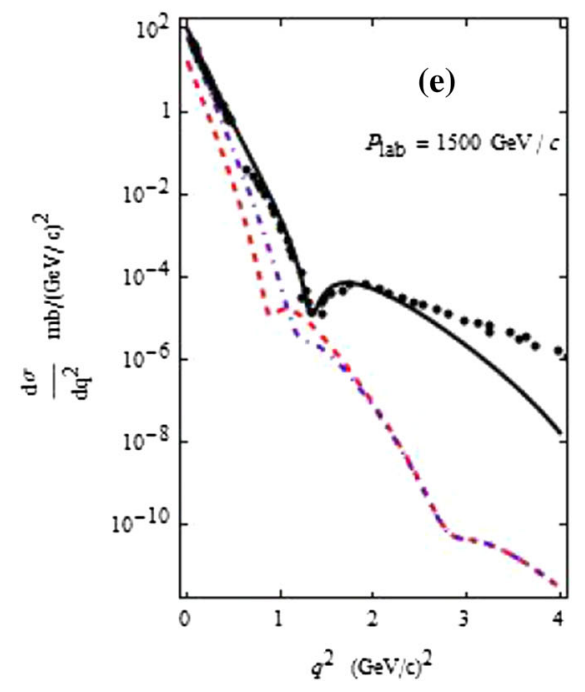


Table 2 The values of radius of quark-quark interaction and gluon quant energy. $r_{t h}$ and $r_{t}$ are the radius of three-quark force and two-quark force, respectively. $E_{2 g}$ and $E_{g}$ are the two-gluon exchange and one-gluon exchange energy

\begin{tabular}{lllllll}
\hline$P_{\text {lap }} \mathrm{GeV} / \mathrm{c}$ & $\beta \mathrm{fm}^{2}$ & $\gamma \mathrm{fm}^{2}$ & $r_{t h}=\sqrt{\gamma} \mathrm{fm}$ & $r_{t}=\sqrt{2 \beta} \mathrm{fm}$ & $E_{2 g} \mathrm{MeV}$ & $E_{g} \mathrm{MeV}$ \\
\hline 200 & 0.274 & 0.14 & 0.37 & 0.74 & 532.15 & 266.07 \\
290 & 0.205 & 0.10 & 0.32 & 0.64 & 614.99 & 307.49 \\
500 & 0.304 & 0.15 & 0.39 & 0.78 & 503.44 & 251.72 \\
1070 & 0.274 & 0.13 & 0.37 & 0.74 & 539.10 & 269.55 \\
1500 & 0.274 & 0.13 & 0.37 & 0.74 & 539.10 & 269.55 \\
Average & 0.266 & 0.13 & 0.364 & 0.738 & 543.39 & 271.695 \\
\hline
\end{tabular}

where $c_{0}$ and $B_{0}$ are dimensionless constants. Therefore, in the calculations of $p-p$ elastic scattering differential cross section we will use the equation [35]

$\frac{d \sigma}{d q^{2}}=\pi\left|T_{0}(q)+F(q)\right|^{2}$.

For the total cross section we will use

$\sigma_{t}(s)=4 \pi \operatorname{Im}\left(T_{0}(0)+F(0)\right)$.

The ratio $\rho_{p p}$ of real part to imaginary part of the elastic scattering amplitude in the forward direction is given by

$\rho_{p p}=\frac{\operatorname{Re}\left(T_{0}(0)+F(0)\right)}{\operatorname{Im}\left(T_{0}(0)+F(0)\right)}$.

$\alpha_{0}=\alpha R_{0}^{2}$

\section{Results and discussion}

Since the experimental evaluation of quark-quark scattering amplitude parameters is not available and for simplicity we will assume that $A_{0 j k}=A_{0}, \varepsilon_{j k}=\varepsilon$ and $\beta_{0 j k}=\beta_{0}$ for all $j$ and $k$. Also, we assume that $A_{j(k l)}=A_{(j m) k}=A$ and $B_{j(k l)}=B_{(j m) k}=B$ for all $j, k, l$ and $m$. Thus, with these approximations the total proton-proton optical phase shift $\chi_{\text {opt }}$ becomes,

$$
\begin{aligned}
\chi_{o p t}\left(\boldsymbol{b}_{0}\right)= & \chi_{o p t}^{t}\left(\boldsymbol{b}_{0}\right)+\chi_{o p t}^{t h}\left(\boldsymbol{b}_{0}\right) \\
= & \frac{9 \alpha_{0} A_{0}(i+\varepsilon)}{12 \pi\left(2+3 \alpha_{0} \beta_{0}\right)} \\
& \exp \left\{-\left[3 \alpha_{0} /\left(4+6 \alpha_{0} \beta_{0}\right)\right] \boldsymbol{b}_{0}^{2}\right\} \\
& +\frac{108 \alpha_{0}^{2} \gamma_{0}^{2}(i A-B)}{5+10 \alpha_{0} \gamma_{0}+3 \alpha_{0}^{2} \gamma_{0}^{2}} \\
& \times \exp \left\{-\frac{3 \alpha_{0}\left(2+\alpha_{0} \gamma_{0}\right)}{5+10 \alpha_{0} \gamma_{0}+3 \alpha_{0}^{2} \gamma_{0}^{2}} \boldsymbol{b}_{0}^{2}\right\}
\end{aligned}
$$

To obtain a good fit in the forward direction of proton-proton elastic scattering differential cross section we will take into account the pion-cloud effect. In Ref. [31] this pion-cloud effect is represented by an additional phenomenological term which takes the form

$$
\boldsymbol{T}_{\boldsymbol{0}}(\boldsymbol{q})=i R_{0}^{2}(s) B_{0} \exp \left\{-c_{0} q_{0}^{2} / 2\right\}
$$

The $\mathrm{p}-\mathrm{p}$ elastic scattering differential cross section at the laboratory momenta 200, 290, 500, 1070 and $1500 \mathrm{GeV} / \mathrm{c}$ is calculated including two-gluon three-quark force and pion cloud effect. The experimental data are taken from [5] for 200 GeV/c and from [6-9] for 290, 500, 1070 and $1500 \mathrm{GeV} / \mathrm{c}$. The values of the quark-quark parameters which are used in the calculations are given in Table 1. The results of the differential cross section are given in the Fig. 1. It is clear, from the Fig. 1, that the optical limit approximation with two-quark force only cannot describe the differential cross section at all the used energies, dashed curves in the figure. The values of theoretical results are clearly below the experimental data. This may be due, partially, to the absence of the multi-scattering terms in this approximation. The inclusion of pion-cloud effect leads to an improvement of the results in the forward direction. We obtained a good fit with the experimental data up to $q^{2} \approx 3(\mathrm{GeV} / \mathrm{c})^{2}$ by including the three-quark force effect.

These results are obtained with quark-quark radius of interaction (in average) is $r_{t}=\sqrt{2 \beta}=0.738 \mathrm{fm}$ and the three-quark force radius (in average) is $r_{t h}=\sqrt{\gamma}=$ $0.364 \mathrm{fm}=(1 / 2) r_{t}$, see Table 2 . The obtained value for strong force radius between two quarks inside the proton is consistent with the measured values of the proton radius, $r_{p}=0.84-0.87 \mathrm{fm}[42]$. 
Fig. 2 Same as Fig. 1. The solid and dashed curves represent the results of (two-+ three-quark forces+ pion-cloud) effects of the Fig. 1 and the results of the average values of the parameters in Table 1, respectively
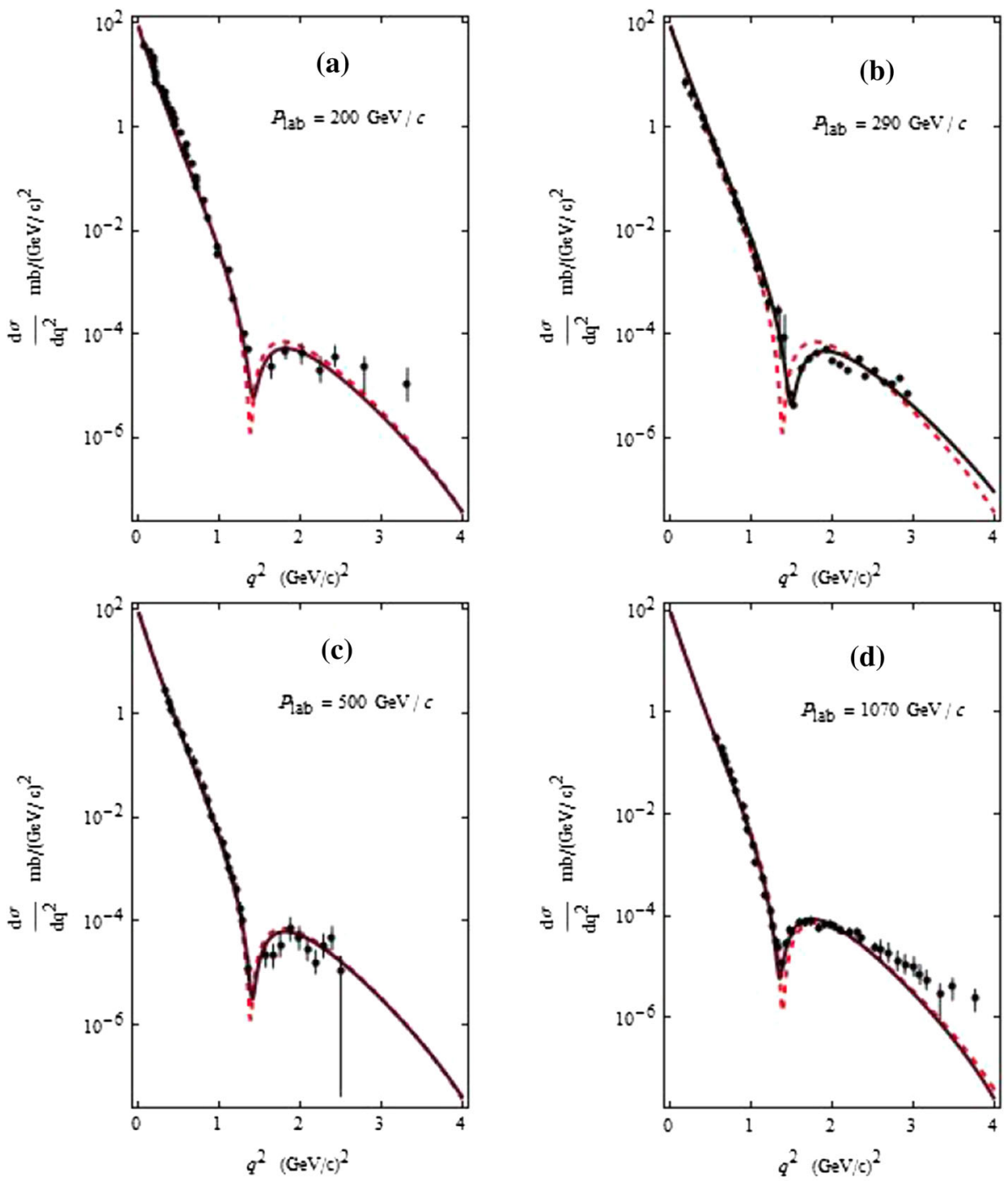
Table 3 Total cross section with (two-quark force, $\sigma^{t}{ }_{t p p}$ ), with (twoquark force +pion cloud, $\sigma^{t+c} t_{t p p}$ ) and with (two-quark force +pion cloud+three-quark force, $\sigma^{t+c+t h}{ }_{t p p}$ ). The ratio of real to imaginary parts of $\mathrm{p}-\mathrm{p}$ elastic scattering amplitude in the forward direc- tion with(two-quark force, $\rho^{t}{ }_{p p}$ ), with (two-quark force +pion cloud, $\rho_{p p}^{t+c}$ ) and with (two-quark force + pion cloud+three-quark force, $\left.\rho^{t+c+t h}{ }_{p p}\right)$. The experimental data are taken from $[39,40]$

\begin{tabular}{lllllllll}
\hline$P_{\text {lap }}(\mathrm{GeV} / \mathrm{c})$ & $\sigma_{t p p}^{t} \mathrm{mb}$ & $\sigma_{t p p}^{t+c} \mathrm{mb}$ & $\sigma_{p p}^{t+c+t h} \mathrm{mb}$ & $\sigma_{t p p}^{\exp } \mathrm{mb}$ & $\rho_{p p}^{t}$ & $\rho_{p p}^{t+c}$ & $\rho_{p p}^{t+c+c h}$ & $\rho_{p p}^{\exp }$ \\
\hline 200 & 15.33 & 30.01 & 38.81 & 38.81 & -0.1434 & -0.0732 & -0.0529 & $-0.038 \pm 0.014$ \\
290 & 15.33 & 31.87 & 39.28 & 39.28 & 0.0465 & 0.0224 & -0.0202 & $0.003 \pm 0.014$ \\
500 & 15.94 & 30.91 & 40.14 & 40.14 & 0.1451 & 0.0749 & 0.0456 & $0.037 \pm 0.006$ \\
1070 & 16.75 & 32.42 & 41.79 & 41.79 & 0.2323 & 0.1200 & 0.0591 & $0.062 \pm 0.011$ \\
1500 & 16.74 & 33.72 & 42.50 & 42.50 & 0.2412 & 0.1197 & 0.0660 & $0.076 \pm 0.009$ \\
\hline
\end{tabular}

From the uncertainty relation of time and energy $\Delta t \Delta E \geq$ $\hbar$ and using the obtained radius of quark-quark interaction we get the quant energy representing the mediator of the force between two quarks (gluon) $E_{g} \approx 271.695 \mathrm{MeV}$ in average. $E_{2 g}=2 E_{g} \approx 543.39 \mathrm{MeV}$, in the Table 2, represent twogluon exchange in the case of three-quark force. We must note that the values of each of quantities $r_{t}, r_{t h}, E_{g}$ have the same order at different considered energies. Therefore, we take the average of all cases.

To confirm the obtained radius of the two- and three quark forces and other parameter values, the $\mathrm{p}-\mathrm{p}$ total cross section $\sigma_{t p p}$ and the ratio $\rho_{p p}$ are calculated. The results are given in the Table 3. The contributions of pion-cloud and three-quark force are very clear and are important for the two quantities. Inclusion of pion cloud improves the results, but with threequark force the results match with experimental data for the total cross section and for the ratio the results are in good agreement with the experimental data.

Since the rest mass of three quarks inside the proton is considered a small part of proton mass, and the other part of the proton mass is coming from the field particles inside the proton (gluons) and the field particle of the external strong field of proton $(\pi-$ meson $)$, we will define the residual energy of proton as follows

$$
\begin{aligned}
E_{\text {res }}= & \text { proton mass }-(\text { mass of three quarks } \\
& + \text { sum of gluons of two }- \text { quark interactions }) \\
= & m_{p}-\left(\text { mass of three quarks }+E_{\text {guи }}\right. \\
& \left.+E_{\text {gud }}+E_{\text {gud }}\right)
\end{aligned}
$$

Assuming that $E_{\text {guu }}=E_{\text {gud }}=E g=264.22 \mathrm{MeV}$ in the average (the value $307.49 \mathrm{MeV}$ is excluded) and since $m_{p}=$ 938.27 MeV and the rest mass of three valence quarks of proton together (in average) is about $9.95 \mathrm{MeV}$ [43] we have,

$$
\begin{aligned}
E_{\text {res }} & =938.27-(9.95+3 \times 264.22)=135.66 \mathrm{MeV} \\
& \approx \text { the mass of } \pi-\text { meson } m_{\pi} \\
& \approx \text { the mass of particle of strong field of proton } .
\end{aligned}
$$

Since $E_{\pi} \approx \frac{1}{2} E_{g}$, this interprets the absence of the quark outside of proton, especially if we note that the three-quark force, with small radius $r_{t h}=0.364 \mathrm{fm}$, takes an important place inside the core of the proton of radius $0.4 \mathrm{fm}$ and each quark is tied with the other two quarks by two gluons and $E_{\pi} \approx \frac{1}{4} E_{2 g}$.

By using the average values of the parameters in the Table 1 to calculate the elastic scattering differential cross section, Fig. 2, we have approximately the same results as in the Fig. 1 with a little difference at the first minimum. Therefore, we can consider that two-quark force, three-quark force and pion-cloud parameters are slightly dependent on energy.

\section{Conclusions}

In conclusion, taking into account the pion-cloud and threequark force effects, by using the optical limit approximation a good fit with the experimental data of $\mathrm{p}-\mathrm{p}$ elastic scattering differential cross section up to $q^{2} \approx 3(\mathrm{GeV} / \mathrm{c})^{2}$, total cross section and the ratio of real to imaginary parts of elastic scattering amplitude in the forward direction is obtained at the laboratory momenta 200, 290, 500, 1070 and $1500 \mathrm{GeV} / \mathrm{c}$. The radii of two-quark interaction $r_{t}$ and three-quark force $r_{t h}$ are calculated. The quant energy representing gluon $E_{g}$ and the mass of the field particle of strong interaction outside the proton $m_{\pi}$ are evaluated.

Open Access This article is distributed under the terms of the Creative Commons Attribution 4.0 International License (http://creativecomm ons.org/licenses/by/4.0/), which permits unrestricted use, distribution, and reproduction in any medium, provided you give appropriate credit to the original author(s) and the source, provide a link to the Creative Commons license, and indicate if changes were made.

Funded by $\mathrm{SCOAP}^{3}$.

\section{References}

1. D. Figueiredo (2013). arXiv:1304.7695v1

2. G. Antchev et al. (TOTEM Collaboration), EPL 96, 21002 (2011)

3. G. Antchev et al. (TOTEM Collaboration), Phys. Rev. Lett. 111, 012001 (2013)

4. G. Antchev et al. (TOTEM Collaboration), EPL 101, 21002 (2013) 
5. J.L. Hartmann et al., Phys. Rev. Lett. 39, 975 (1977)

6. A. Bömh et al., Phys. Lett. B 49, 491 (1974)

7. A. Breakstone et al., Phys. Rev. Lett. 54, 2180 (1985)

8. H. De Kerret et al., Phys. Lett. B 62, 363 (1976)

9. N. Kwak et al., Phys. Lett. B 58, 233 (1975)

10. P.D.B. Collins, F.D. Gault, Phys. Lett. B 73, 330 (1978)

11. A. Donnachie, H.G. Dosch, P.V. Landshoff, O. Nachtmann, Pomeron Physics and QCD (Cambridge University Press, Cambridge, 2002)

12. O. Nachtmann (2003). arXiv:hep-ph/0312279

13. C. Bourrely, J. Soffer, T.T. Wu, Eur. Phys. J. C 28, 97 (2003)

14. S. Ostapchenko (2010). arXiv:1003.0196v1

15. P. Desgrolard, M. Giffon, E. Martynov, E. Predazzi, Eur. Phys. J. C 16, 499 (2000)

16. T. Csörgő, F. Nemes, Int. J. Mod. Phys. A 29, 1450019 (2014)

17. V.A. Okorokov, S.D. Campos, Int. J. Mod. Phys. A 32, 1750175 (2017)

18. M. Wei-hsing, H. Chao-hui, W. De-an, Nucl. Phys. A 496, 729 (1989)

19. H. Chao-hui, W. De-an, M. Wei-hsing, Nucl. Phys. A 518, 717 (1990)

20. H.X. Zhong, Phys. Rev. C 51, 2700 (1995)

21. S.V. Goloskokov (1998). arXiv:hep-ph/9809578v1

22. S.V. Goloskokov, P. Kroll (2000). arXiv:hep-ph/9807529v2

23. S. Bondarenko, E. Levin, Eur. Phys. J. C 51, 659 (2007)

24. M. Broilo, E.G.S. Luna, M.J. Menon (2018). arXiv:1803.06560v2

25. S. Bondarenko, E. Levin, J. Nuiri, Eur. Phys. J. C 25, 277 (2002)

26. Y.M. Shabelski, A.G. Shuvaev, JHEP 1411, 023 (2014). arXiv:1406.1421 [rhep- ph]
27. Y.M. Shabelski, A.G. Shuvaev, Eur. Phys. J. C 78, 497 (2018)

28. R.J. Glauber, Lectures on Theoretical Physics, ed. by W.E. Brittin, L.C. Dunham, vol. 1 (Interscience, New York, 1959), p. 315

29. Y. Takada, Prog. Theor. Phys. 39, 1536 (1968)

30. M. Tanimoto, S. Wakaizumi, Prog. Theor. Phys. 52, 1000 (1974)

31. S. Wakaizumi, Prog. Theor. Phys. 60, 1040 (1978)

32. M.A. Hassan, A.A.R. Yousef, S.S.A. Hassan, Aust. J. Phys. 48, 821 (1995)

33. M. Kuroda, H. Miyazawa, Prog. Theor. Phys. 50, 569 (1973)

34. V. Franco, Phys. Rev. C 35, 1328 (1987)

35. M.A. Hassan, T.N.E. Salama, I.M.A. Tag El-Din, J. Phys. G Nucl. Part. Phys. 28, 33 (2002)

36. M.M. Islam, R.J. Luddy, A.V. Prokudin, Int. J. Mod. Phys. A 21, 1 (2006)

37. M.A. Hassan, T.N.E. Salama, Z.S. Hassan, Phys. Rev. C 63, 04606 (2001)

38. M.A. Hassan, T.N.E. Salama, Z.S. Hassan, H.E. Awd-Allah, J. Phys. G Nucl. Part. Phys. 40, 065102 (2013)

39. M.M. Block, R.N. Cahn, Proc. 2nd Int. Conf. on Elastic and Diffractive Scattering, ed. by K. Goulianos (Kim Hup Lee Printing Co. Pte. Ltd., Singapore, 1987), p. 85

40. M.M. Block, B. Margolis, P. Valin, Proc. 2nd Int. Conf. on Elastic and Diffractive Scattering, ed. by K. Goulianos (Kim Hup Lee Printing Co. Pte. Ltd., Singapore, 1987), p. 107

41. J. Dias de Deus, Nucl. Phys. B 54, 23 (1973)

42. R. Pohl et al., Nature 466, 213 (2010)

43. K. Nakamura et al. (Particle Data Group), J. Phys. G Nucl. Part. Phys. 37, 075021 (2010) 